\title{
Analysis of rail potential and stray current in MVDC railway electrification system
}

\author{
Salman Aatif ${ }^{1,2}$ (D) Haitao $\mathrm{Hu}^{1} \cdot$ Fezan Rafiq $^{1,3} \cdot$ Zhengyou He $^{1}$
}

Received: 20 March 2021/Revised: 9 June 2021/Accepted: 10 June 2021 / Published online: 13 July 2021

(C) The Author(s) 2021

\begin{abstract}
In contrast to the conventional direct current railway electrification system (DC-RES), the medium voltage direct current (MVDC)-RES is considered promising for long-distance high-speed corridors. In the MVDC-RES, traction substations (TSSs) are placed much farther and train loads are much heavier than in the conventional DC-RES. Hence, the MVDC-RES brings a drastic change in catenary voltage, TSS spacing, and train loading, which affects rail potential and stray current. In this connection, this work performs some significant quantitative analysis of rail potential and stray current in the MVDC-RES environment. An MVDC simulation model is proposed and different grounding schemes are analyzed for a single-train and two TSSs scenario as well as for a multi-train multi-TSS scenario. According to the simulation and analysis, the maximum values of rail potential and stray current at MVDC-RES distances and the maximum safe distance between adjacent TSSs are determined.
\end{abstract}

Salman Aatif

salmanatif@uetpeshawar.edu.pk

Haitao $\mathrm{Hu}$

hht@swjtu.edu.cn

Fezan Rafiq

fezan@neduet.edu.pk

Zhengyou $\mathrm{He}$

hezy@swjtu.edu.cn

1 School of Electrical Engineering, Southwest Jiaotong University, Chengdu 610031, China

2 Department of Electrical Engineering (Bannu Campus), University of Engineering and Technology Peshawar, Peshawar, Pakistan

3 Department of Electrical Engineering, NED University of Engineering and Technology Karachi, Karachi, Pakistan
Keywords Railway electrification - Medium voltage direct current (MVDC) railways - Rail potential - Stray current · Stray corrosion

\section{Introduction}

Railway systems have emerged as a key means of transportation since the nineteenth century. The modern highspeed traction railways are more fast, reliable, and environment-friendly means of transportation. For greater benefits, researchers are exploring new avenues of efficient and enhanced railway electrification operations. Inspired by the applications of high voltage direct current (HVDC) technology in conventional power transmission systems in recent history, some scholars have proposed the idea of medium voltage direct current (MVDC) railway electrification system (RES) for long-distance high-speed railways (HSRs). In this connection, the basic architecture, advantages, and voltage control schemes for MVDC railway are further discussed [1-3]. As the MVDC-RES causes less reactance, the distance between the traction substations (TSSs) can be further increased; this brings the benefit of decreased capital and operational cost of the TSSs [4]. Moreover, the catenary system becomes a continuous traction line and the train loads can be shared by all the TSSs in the system [5].

In DC railways, the running rail is used as a return conductor; the return current flowing toward the TSSs in the rails causes a potential difference between the rail and earth, which is known as track to earth voltage or rail potential. This voltage causes stray current to leak into the 
earth, which causes corrosion to the third party as well as to the railway's own infrastructure [6]. Stray current has a direct relationship to the distance between TSS and train load (load current), and is inversely proportional to rail-toearth resistance $[7,8]$ :

$I_{\mathrm{s}} \propto \frac{I_{\mathrm{L}} l}{R_{\mathrm{g}}}$,

where $I_{\mathrm{S}}$ is stray current, $I_{\mathrm{L}}$ is rail current caused by the train load, $l$ is the distance between the considered location and TSS, and $R_{\mathrm{g}}$ is the rail-to-earth resistance.

In an MVDC-RES, the distance between adjacent TSSs is many folds (almost 25-35 times) as compared to the conventional urban DC railways [3,5]. Similarly, the train load is also heavier (approximately 3-4 times) than conventional urban DC railways [2]. However, the catenary voltage in the MVDC-RES is quite higher than in the conventional urban DC railways [4]. The authors have discussed the stray current in MVDC-RES in [4]; the aim of this paper is limited to the effectiveness of the integration topology of secondary energy sources for decreasing rail potential and stray current at mid-sections. It is unable to provide the information of stray current profile for different track lengths under different grounding schemes in a multi-train, multi-TSS scenario. Similarly in [9], authors have performed the analysis of rail potential and stray current for $9 \mathrm{kV}$ system. However, the paper considered several other constraints, which may have restricted the full-scale analysis of rail potential and stray current.

According to the aforementioned discussion, there is of vital importance to quantify the rail potential and stray current in the MVDC-RES. In this regard, the proposed work presents a detailed analysis of rail potential and stray current in the MVDC-RES with different lengths of the traction lines, load scenarios, and grounding schemes. The main features of this paper include: (1) MVDC-RES environment, (2) rail potential and stray current under different grounding schemes, (3) proposed simulation platform for the analysis of rail potential and stray current, and (4) simulations for identifying safe operation distances between adjacent TSSs in MVDC railways.

\section{Rail potential and stray current under different TSS grounding schemes}

\subsection{MVDC railway electrification system}

The basic layout of an MVDC-RES is provided in Fig. 1 [2]. To understand the real benefits of the DC technology in the MVDC-RES, the authors [4] have suggested a $24 \mathrm{kV}$ electrification scheme. The TSSs consist of a transformer and multilevel AC-DC converters. The catenary system is

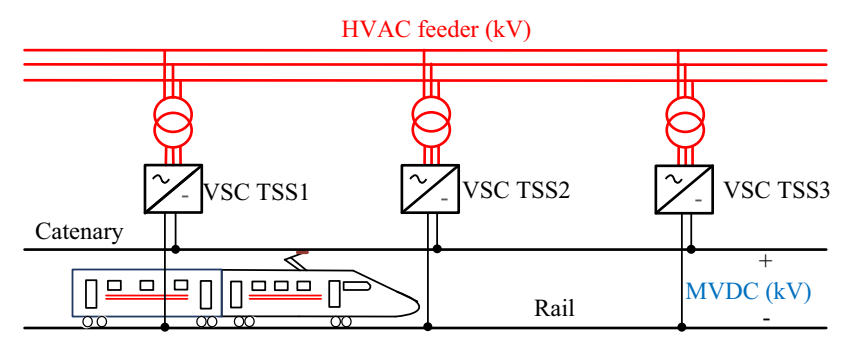

Fig. 1 The layout of MVDC railway electrification system

the same as the one used in urban DC railways. The detailed architecture and advantages of the system are presented in $[2,3]$.

\subsection{Rail potential and stray current}

In a DC traction system, the return current from locomotives is fed back to the negative terminal of the TSS through the running rails, which causes rail potential and stray current. Higher values of rail potential cause electrocution, which is hazardous for humans as well as for animals in the vicinity of the track. For safety purposes, the maximum value of rail-to-earth voltage $\left(V_{\mathrm{rg}}\right)$ must be less than $90 \mathrm{~V}$ [10]. The safe operating values of rail-to-earth voltage for different sections, i.e., at TSSs, workshops, and along the track, are provided in [10]. The stray current leaking from the rail moves within the earth toward the negative terminal (in grounded scheme) or toward the area of the track having lower potential (in floating rail scheme) [7]. In both the cases, this stray current also flows through metallic objects within the earth specifically through buried pipelines that causes corrosion [11]. It is also noteworthy that corrosion occurs at the anodic region of the buried pipelines: the region from where the current leaves the buried metallic objects and enters the earth again, as shown in Fig. 2. To avoid corrosion, the average value of stray current $\left(I_{\mathrm{s}}\right)$ must be less than $2.5 \mathrm{~mA} / \mathrm{m}[11,12]$.

\subsection{Traction substation grounding schemes in DC railways}

The main objective of a grounding scheme in a DC-RES is to fulfill two mutually contradictory requirements: to minimize rail potential and to decrease the stray current, for the protection of humans and equipments [13]. In the modern DC-RES, these requirements are met by integrating a combination of grounding schemes [13, 14]. Some of the common grounding schemes used in the DC traction system are grounded scheme, floating scheme (ungrounded scheme), and diode or thyristor grounded scheme.

In a grounded scheme, the negative terminal of the TSS is grounded without any intentional resistance as shown in 


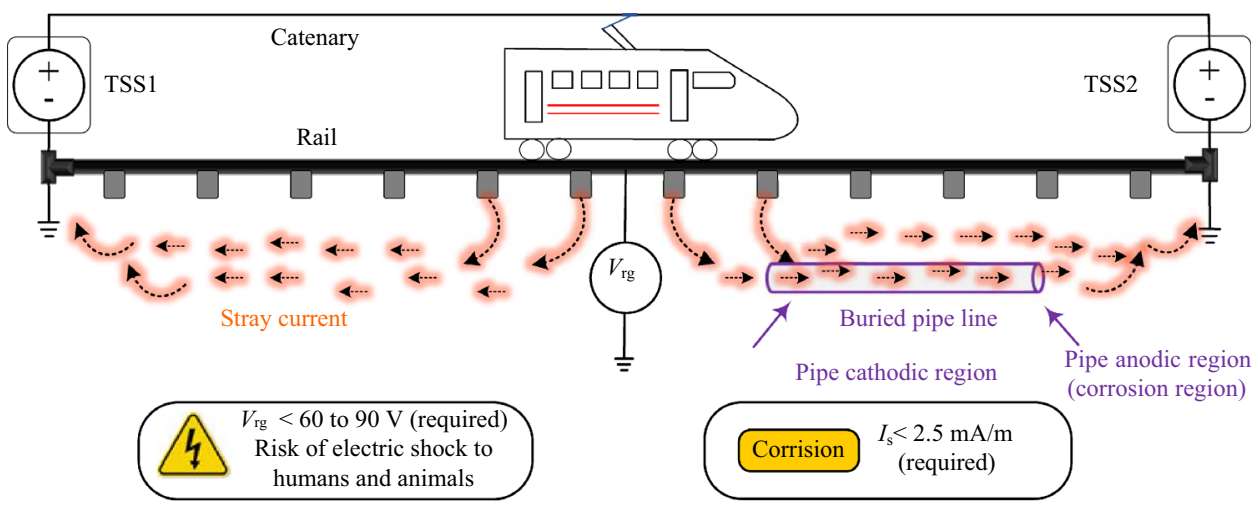

Fig. 2 Earth grounded scheme in DC electrified railways

Fig. 2. Such a scheme reduces rail potential; however, it increases the issue of corrosion as more stray current leaves the rail in the presence of earth potential. This stray current flows within the earth, parallel to traction line, toward the grounding location of the TSS. However, the scheme causes higher stray current, but reduces the rail potential, which that lowers the risk of electric shock and damage to the equipment at TSSs.

In the floating scheme, the negative terminal of the TSS is not grounded and left floating. The stray current leaves the rail at a higher rail-to-ground voltage (near the train) and reenters the rail at some lower potential section of the track as shown in Fig. 3.

Such a scheme reduces the stray current up to one-fourth produced in the grounded scheme [15], although the scheme reduces stray current while increases the rail potential, more specifically near the TSSs [13]. The situation may become more hazardous if a greater number of trains turn into acceleration mode simultaneously.

The above-stated drawback of the floating system can be ratified through a diode or thyristor grounding scheme [13]. In a diode grounding scheme, the negative terminal of the TSS is grounded through a diode as shown in Fig. 4.

Under normal rail potential, the diode works as an off switch, and the rail is insulated from the ground; hence, the scheme becomes a floating one. Conversely, if the rail potential increases to a critical higher level as compared to ground, the diode will turn to on to ground the negative terminal of the TSS. This causes the stray current to flow through the earth until the rail potential returns to the safe value.

Analysis of rail potential and stray current in conventional DC railways has been extensively studied [16-19]. However, their behavior and quantitative analysis in new MVDC-RES requires an in-depth analysis on a realistic simulation model at an MVDC voltage level under different grounding schemes. For this purpose, a simulation model is proposed and discussed in next section.

\section{Simulation model for rail potential and stray current}

In [20], a fixed and a variable traction resistance models are presented. The fixed resistance model can only provide rail potential and stray current results for static train locations, where the variable resistance model can provide results for moving train scenarios as well. In [21], a modified converter circuit is used to achieve a variable rail network resistance circuit. It is also noteworthy that the train load is a constant power load with dynamic tractive effort. Hence, the stable operation with a variable resistance network is

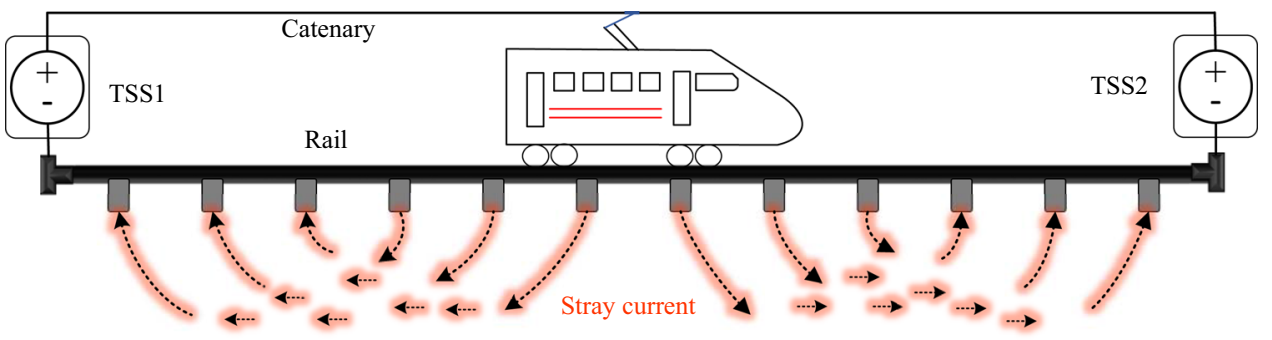

Fig. 3 Floating scheme in DC electrified railways 


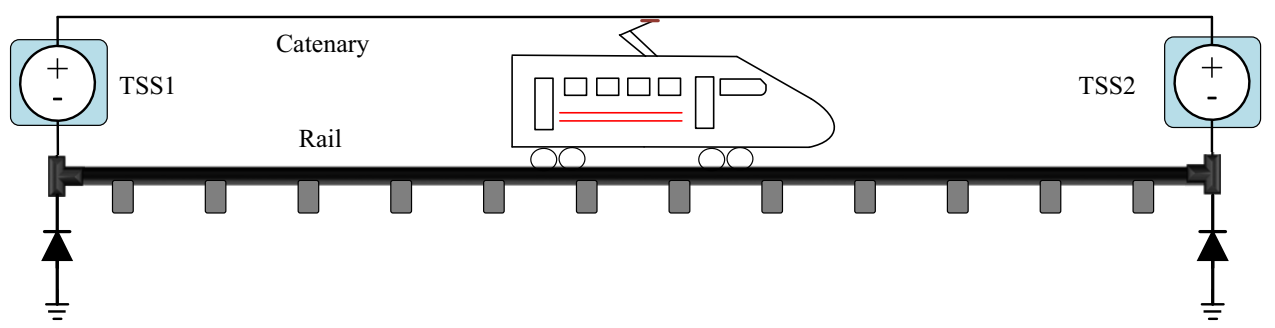

Fig. 4 Diode grounded scheme in DC electrified railways

also a challenge [22-24]. Modeling of a variable resistance network can be achieved through a combination of dependent voltage source and resistance ramp function; however, using such a scheme with train load models also brings stability issues.

The simulation model proposed in this paper consists of a fixed resistance network. However, it can perform the simulations for moving train scenarios as well. Each resistor (catenary resistance, rail resistance, and grounding resistance) is controlled with a physical switch, and operated through a program. The proposed simulation model provides the profile of rail potential and stray current for static as well as moving train loads. Moreover, the proposed traction resistance network does not have any dependent sources, converters, or other active devices as used in previous methods; hence, it contributes no stability issues in the system. Figure 5 shows the simulation model used for the measurement of rail potential and stray current in this work.

To perform the moving train simulations, the model consists of several switches to simulate the catenary network, track network, and grounding network. In Fig. 5, $R_{\mathrm{c} j}$ and $S_{\mathrm{cj}}$ represent catenary resistance and catenary switches respectively; $R_{\mathrm{k} j}$ and $S_{\mathrm{k} j}$ track resistance and track switches; $R_{\mathrm{g} j}$ and $S_{\mathrm{g} j}$ grounding resistance and its switches. Each resistance and corresponding switch represents a unit length $(\mathrm{km})$ section of the traction line. For better understanding, a $5 \mathrm{~km}$ traction line is provided in Fig. 5. The complete journey of a moving train starting from TSS1 and ending at TSS2 can be achieved by the proper operation of switches as mentioned in Table 1. The stray current and rail potential at any $j$ th location along the traction line can be easily obtained through the simulations by considering an ampere meter in series, and a voltmeter across the grounding resistance at the desired location, respectively. For understanding the working of simulation model, a case study for finding rail potential and stray current at midsection of the traction line of Fig. 5 is conducted. For monitoring stray current at mid-section, an ampere meter must be connected in series at $R_{\mathrm{g} 3}$ and $R_{\mathrm{g} 8}$. Similarly, the rail potential at mid-section during the whole journey of the train can be monitored by considering a voltmeter across $R_{\mathrm{g} 3}$ and $R_{\mathrm{g} 8}$. It is noteworthy that the state of grounding switch $S_{\mathrm{g} j}$ must be reciprocal to the corresponding track switch $S_{\mathrm{k} j}$. If there is a train journey from left to right, at time intervals T0-T2 from Table 1 , the load current $I_{\mathrm{L} 2}$ flows through mid-section. Hence, the amperemeter and voltmeter at $R_{\mathrm{g} 8}$ provide the values of stray current and rail potential at mid-section. At time interval T3 to T5, load current $I_{\mathrm{L} 1}$ flows through mid-section, and the amperemeter and voltmeter at $R_{\mathrm{g} 3}$ provide the information of stray current and rail potential at mid-section. Modification in the

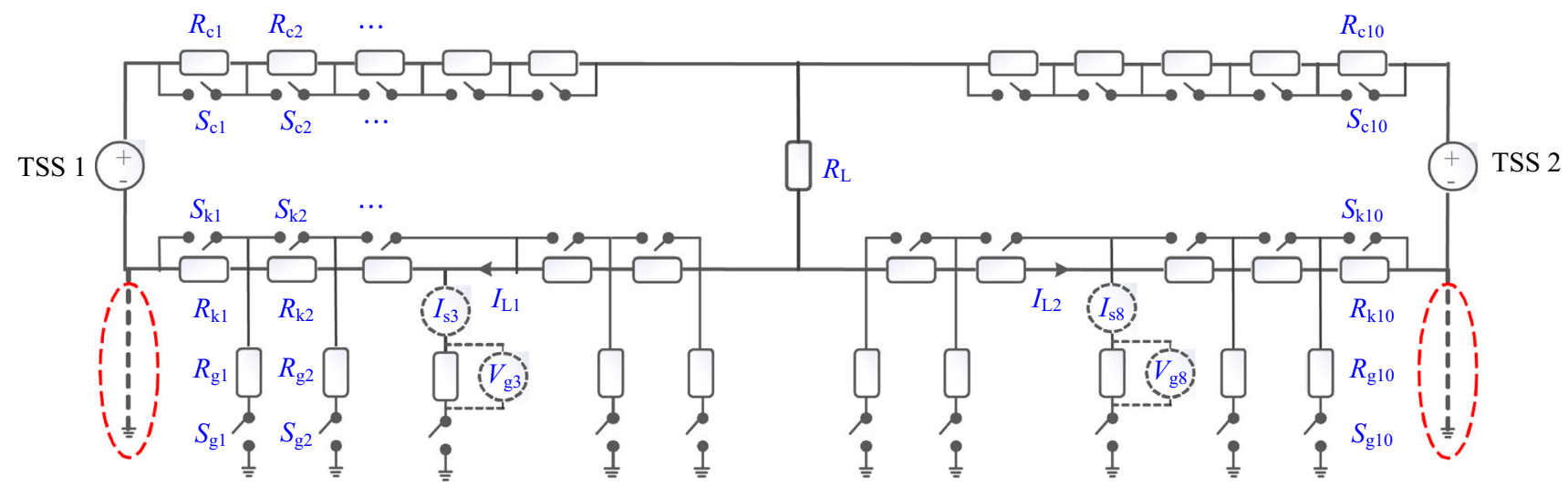

Fig. 5 Simulation model used for the analysis of rail potential and stray current 
Table. 1 The switching sequence for proper operation of the simulation model in Fig. 5

\begin{tabular}{lllllllllll}
\hline Simulation time & $S_{\mathrm{c} 1} / S_{\mathrm{k} 1}$ & $S_{\mathrm{c} 2} / S_{\mathrm{k} 2}$ & $S_{\mathrm{c} 3} / S_{\mathrm{k} 3}$ & $S_{\mathrm{c} 4} / S_{\mathrm{k} 4}$ & $S_{\mathrm{c} 5} / S_{\mathrm{k} 5}$ & $S_{\mathrm{c} 6} / S_{\mathrm{k} 6}$ & $S_{\mathrm{c} 7} / S_{\mathrm{k} 7}$ & $S_{\mathrm{c} 8} / S_{\mathrm{k} 8}$ & $S_{\mathrm{c} 9} / S_{\mathrm{k} 9}$ & $S_{\mathrm{c} 10} / S_{\mathrm{k} 10}$ \\
\hline T0 & 1 & 1 & 1 & 1 & 1 & 0 & 0 & 0 & 0 \\
T1 & 0 & 1 & 1 & 1 & 1 & 1 & 0 & 0 & 0 \\
T2 & 0 & 0 & 1 & 1 & 1 & 1 & 1 & 0 & 0 \\
T3 & 0 & 0 & 0 & 1 & 1 & 1 & 1 & 1 & 0 \\
T4 & 0 & 0 & 0 & 0 & 1 & 1 & 1 & 1 & 0 \\
T5 & 0 & 0 & 0 & 0 & 0 & 1 & 1 & 1 & 0 \\
\hline
\end{tabular}

simulation model for grounded and ungrounded schemes and relations for the verification of the simulation model are discussed in preceding subsections.

\subsection{Grounded scheme simulation model}

The grounding connections mentioned through dotted lines in Fig. 5 must be considered in case of grounded traction scheme. The simulation model used in Fig. 5 provides accurate information of the stray current and rail potential for an ideal grounded system. The estimated value of the stray current can be obtained through Eq. (2) [15]:

$I_{\mathrm{s} j}=\frac{I_{\mathrm{L}} l R_{\mathrm{k}}}{R_{\mathrm{g}}}$,

where $I_{\mathrm{s} j}$ is the stray current at the $j$ th location; $I_{\mathrm{L}}$ is the load current in the rails; $l$ is the distance between the considered location and the TSS; $R_{\mathrm{k}}$ is the resistance of the track per unit length; and $R_{\mathrm{g}}$ is the rail-to-ground resistance. The stray current obtained through Eq. (2) is the total stray current entering the earth at the considered location; this stray current is further divided according to the distance from the two TSSs and flows toward the TSS accordingly. As the train locates at the mid-section and the distance from both the TSSs remains the same, the total stray current obtained from Eq. (2) can be divided by 2 .

\subsection{Floating scheme simulation model}

In case of an ungrounded or floating scheme, there is no grounding connection at the TSS. Conversely, to the grounded traction scheme, the phenomena of stray current and rail potential are more complex in floating traction system. The stray current and rail potential at any $j$ th location along the traction line in floating scheme can be estimated by [12]

$I_{\mathrm{s} j}=0.5 \times \frac{I_{\mathrm{L}}}{l_{\mathrm{c}}} \times\left[1-\mathrm{e}^{-\left(\frac{l}{l_{\mathrm{c}}}\right)}\right]$,
$V_{\mathrm{g} j}=0.5 \times I_{\mathrm{L}} \times R_{\mathrm{c}} \times\left[1-\mathrm{e}^{-\left(\frac{l}{l_{\mathrm{c}}}\right)}\right]$,

where $l_{\mathrm{c}}$ is the characteristic length of the rail and can be evaluated through Eq. (5); $R_{\mathrm{c}}$ is the characteristic resistance of the running rail and can be evaluated through Eq. (6):

$l_{\mathrm{c}}=\frac{1}{\sqrt{R_{\mathrm{k}} / R_{\mathrm{g}}}}$,

$R_{\mathrm{c}}=\frac{1}{\sqrt{R_{\mathrm{k}} \times R_{\mathrm{g}}}}$.

\subsection{Simulation model for train load}

The aim of this work is to analyze the full-scale or maximum possible rail potential and stray current. The considered train load is moving with the maximum speed, having maximum tractive effort, and thus utilizing maximum power throughout the journey. This makes the constant power train load a perfect selection for the analysis. The layout of analog constant power load used in this work is shown in Fig. 6 [24], where $P_{\text {ref }}$ is the reference power; $I_{\mathrm{L}}$ is the load current or train current; $V_{\mathrm{L}}$ is the load voltage; and $I_{\mathrm{L}}^{*}$ is the reference given to the dependent current source. A similar train load is used where the maximum train load is required $[2-4,9]$.

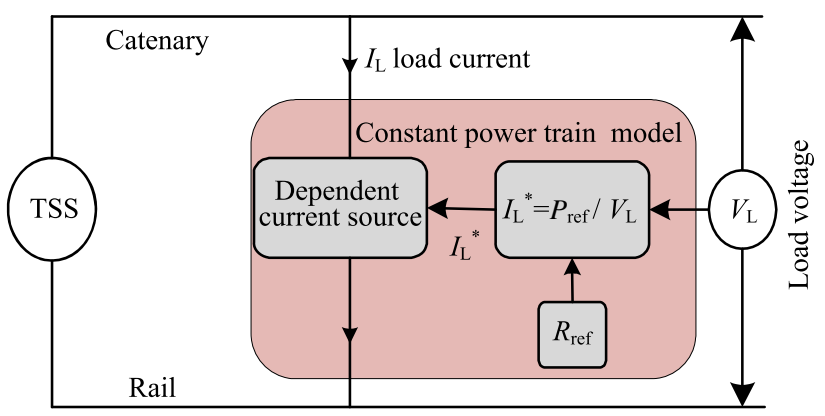

Fig. 6 Layout for constant power train load model 
Table 2 Parameters used in simulation model

\begin{tabular}{ll}
\hline Parameter & Specifications \\
\hline TSSs ratings & $24 \mathrm{kV}, 30 \mathrm{MW}$ \\
Resistance of rail $(\Omega / \mathrm{km})$ & $0.0273 / 2$ \\
Resistance of the catenary & 0.104 \\
$\quad($ contact $/$ messenger wire) $(\Omega / \mathrm{km})$ & \\
Train load (constant power load) $(\mathrm{MW})$ & 8 \\
Rail-to-ground resistance $(\Omega / \mathrm{km})$ & 50 \\
\hline
\end{tabular}

\section{Simulations}

Simulations are performed to analyze rail potential and stray current in an MVDC-RES environment. In the conventional high-speed AC-RES, the distance between the adjacent TSSs is usually $50 \mathrm{~km}$. As in the MVDC-RES the losses are less, the distance between the TSSs can be increased. For simulation purpose, the analysis is performed on a $50 \mathrm{~km}$ track (similar to current AC corridors), and a $75 \mathrm{~km}$ track (similar to prospective MVDC-RES corridors) [3]. Moreover, for detailed analysis, simulations are performed for both grounded and floating electrification schemes in the single as well as multi-train scenario. The parameters used in the simulation model are listed in Table 2.

\subsection{Grounded scheme simulations for $50 \mathrm{~km}$ and $75 \mathrm{~km}$ track}

An extended model of Fig. 5 is used in this section for simulating $50 \mathrm{~km}$ and $75 \mathrm{~km}$ tracks. The switching mechanism is intelligently controlled to simulate the moving train scenarios. The stray current and rail potential profiles are analyzed at specified locations, i.e., at the mid-section and at one-fourth of the section, for the complete journey of the train. If the maximum value of the stray current at any location for the complete journey of the train is less than the maximum allowable average of $2.5 \mathrm{~A} / \mathrm{km}$, then the average value must always be lower.

\section{$4.1 .150 \mathrm{~km}$ grounded track}

Figure 7 depicts the result of rail potential at specific locations, i.e., at the one-fourth and mid-section of the traction line, for the complete journey of the train. In both cases, rail potential is less than the maximum allowable limit of safe rail operation.

The results of stray current at the one-fourth $(12.5 \mathrm{~km})$ of the section and mid-section $(25 \mathrm{~km})$, for the complete journey of the train on $50 \mathrm{~km}$ grounded track, are shown in Fig. 8. It reveals that the stray current reaches its maximum value when the train reaches the location of interest.

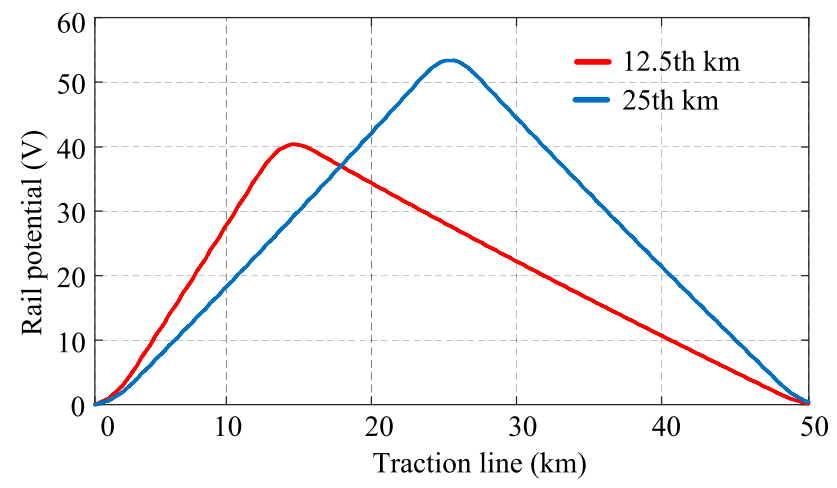

Fig. 7 Rail potential at the mid-section and one-fourth of the section

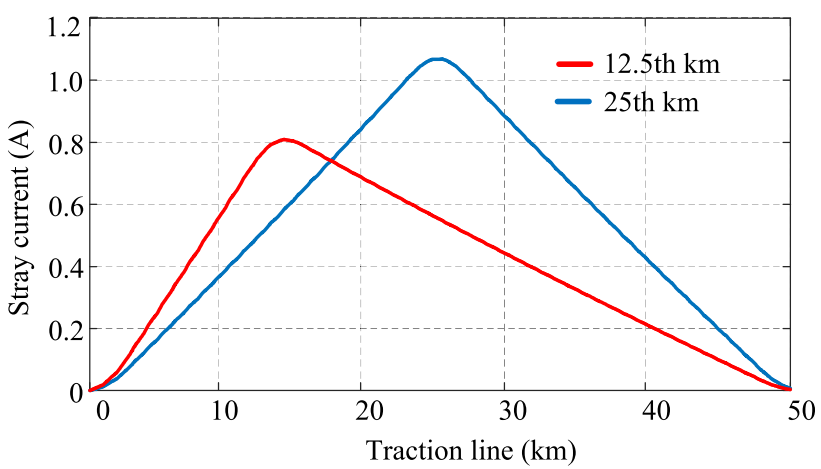

Fig. 8 Stray current monitored for $50 \mathrm{~km}$ grounded track

\subsection{2 $75 \mathrm{~km}$ grounded track}

The rail potential at the one-fourth of the section $(19 \mathrm{~km})$ and mid-section $(37.5 \mathrm{~km})$ for the complete journey of the train is shown in Fig. 9. Results reveal that the rail potential at the mid-section increases up to $77 \mathrm{~V}$,

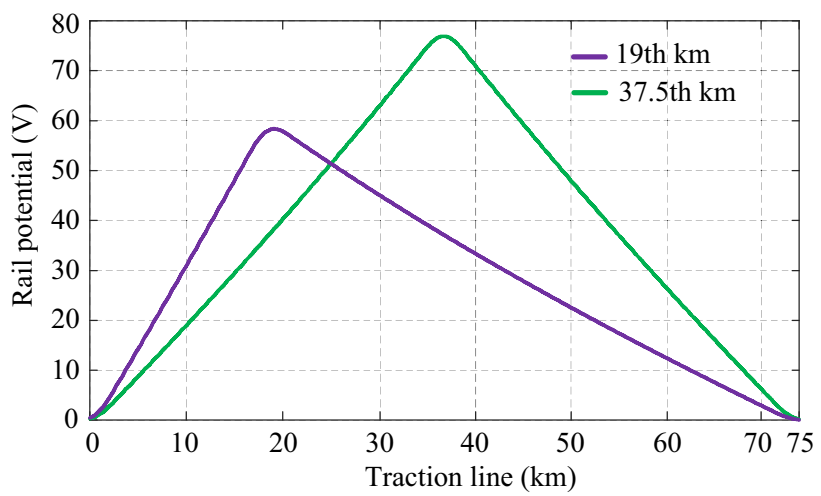

Fig. 9 Rail potential monitored at specified locations on the $75 \mathrm{~km}$ grounded track 


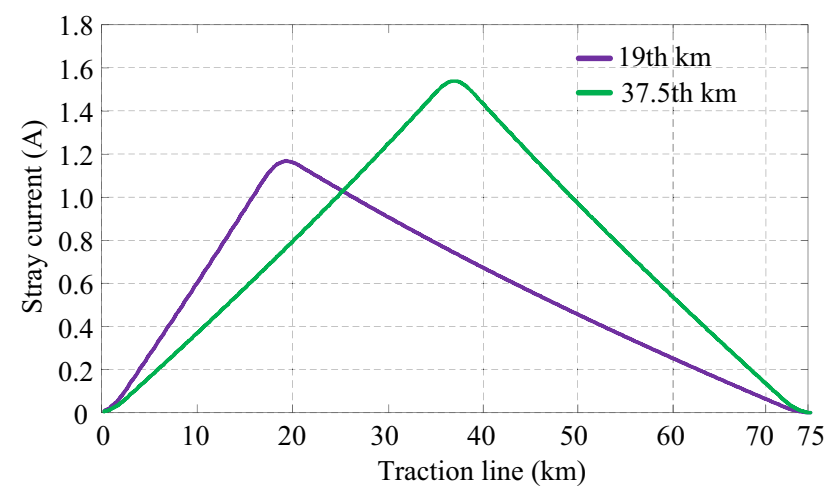

Fig. 10 Stray current monitored at specified locations on the $75 \mathrm{~km}$ grounded track

approaching the maximum safe limits of $90 \mathrm{~V}$. It can be concluded that increasing the distance further between the adjacent TSSs can cause risk of electric shock to humans and animals near the traction line.

The stray currents profile at a distance of the one-fourth of the track $(19 \mathrm{~km})$ and at the mid-section $(37.5 \mathrm{~km})$, for the complete journey of the train, is provided in Fig. 10. The results reveal that the maximum stray current at midsection is still less than that of the average safe value of $2.5 \mathrm{~A} / \mathrm{km}$. Although the current is less than the maximum limit for the case of a single-train operation between adjacent TSSs, two or more trains can increase that value to hazardous levels.

\subsubsection{Verification of simulations}

Simulation results for stray current and rail potential depicted for the grounded system can be verified through Eq. (2). The verification is performed for complete profile of stray current at the one-fourth of $50 \mathrm{~km}(12.5 \mathrm{~km})$. Figure 11 shows a simplified version of Fig. 5.

Given the train direction from left to right, initially when the train is in area A, the track current $I_{2}$ is the only current that causes rail potential and stray current at the one-fourth

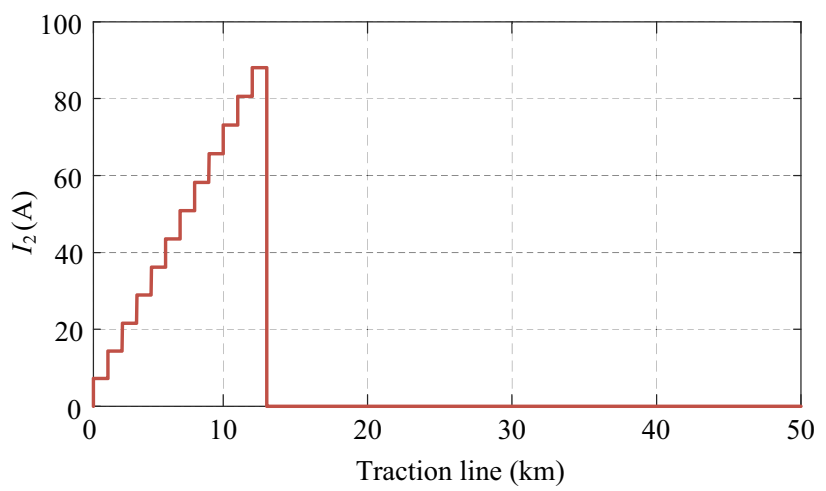

Fig. 12 Track current $I_{2}$ at the 12.5 th $\mathrm{km}$ for train journey in area A

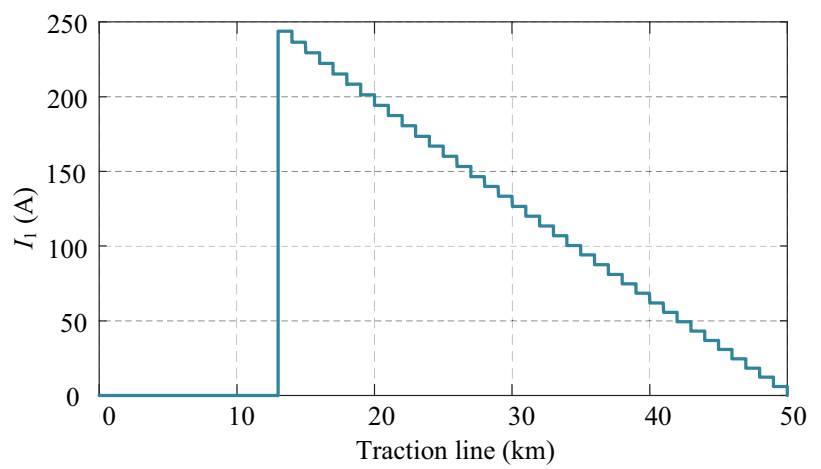

Fig. 13 Track current $I_{1}$ at the 12.5th km for train journey in area B

distance or at the 12.5 th $\mathrm{km}$ resistor. $I_{2}$ can be monitored through an ammeter $A_{a}$ which can be used in Eq. (2) for the estimation of stray current at the 12.5 th $\mathrm{km}$. As the train continues its journey and enters in area $\mathrm{B}$, the track current $I_{1}$ now causes rail potential and stray current at the 12.5 th $\mathrm{km}$. Current $I_{1}$ can be monitored through ammeter $\mathrm{A}_{\mathrm{b}} . I_{1}$ can be used in Eq. (2) for the estimation of stray current at the $12.5 \mathrm{th} \mathrm{km}$ of the track for the train in area B. The portions of track currents $I_{2}$ and $I_{1}$ which cause stray current at the 12.5 th $\mathrm{km}$ are provided in Figs. 12 and 13, respectively.

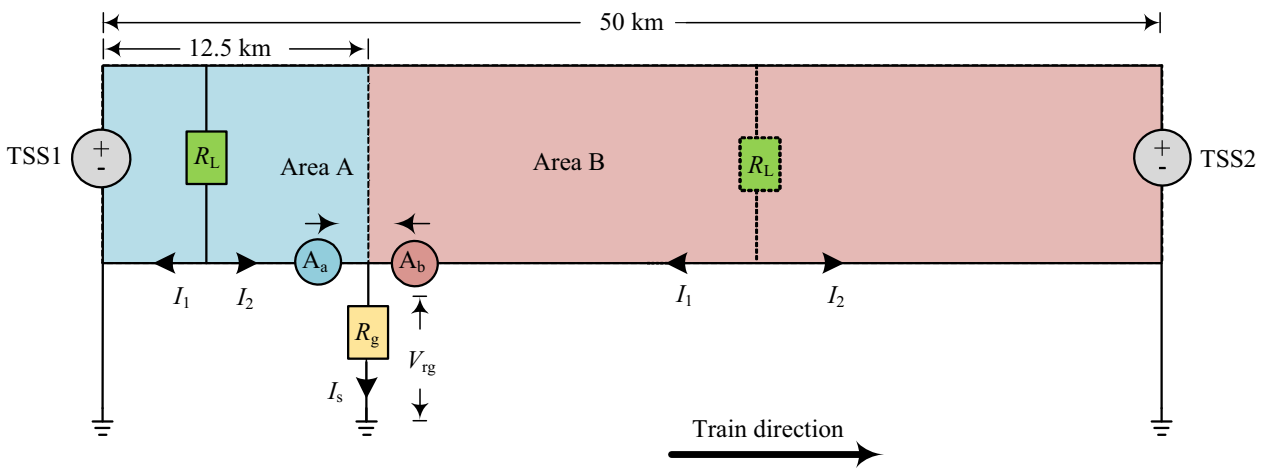

Fig. 11 A simplified model of Fig. 5 for the verification of stray current (50 km grounded track) 
Table 3 Validation of stray currents

\begin{tabular}{llllll}
\hline Train location $(\mathrm{km})$ & $I_{2}(\mathrm{~A})$ & $I_{1}(\mathrm{~A})$ & Length considered $l(\mathrm{~km})$ & Estimated stray current through Eq. (2) $(\mathrm{A})$ & Simulated stray current $(\mathrm{A})$ \\
\hline 5 & 30 & - & 37.5 & 0.31 & 0.29 \\
12.5 & 88 & 244 & $37.5,12.5$ & $0.91,0.84$ & 0.81 \\
25 & - & 164 & 12.5 & 0.566 & 0.59 \\
40 & - & 65 & 12.5 & 0.224 & 0.26 \\
\end{tabular}

Considering the values of track currents from the above figures, the stray current at the 12.5 th $\mathrm{km}$ is estimated through Eq. (2), for the train positioned at 5, 12.5, 25, and $40 \mathrm{~km}$. The results are compared with the simulated values of Fig. 8, as shown in Table 3. It can be observed from Table 3 that the estimated values are consistent with the simulation results.

\subsection{Floating scheme simulations for $50 \mathrm{~km}$ and $75 \mathrm{~km}$ tracks}

As discussed earlier, the phenomena of stray current and rail potential are more complex in a floating scheme. For simulations, $50 \mathrm{~km}$ and $75 \mathrm{~km}$ tracks are considered, which represent the distances between adjacent TSSs for $25 \mathrm{kV}$ AC-RES and $24 \mathrm{kV}$ MVDC-RES, respectively.

\subsection{1 $50 \mathrm{~km}$ floating track}

Simulations are performed to find the rail potential and stray current at the one-fourth $(12.5 \mathrm{~km})$ and at mid-section $(25 \mathrm{th} \mathrm{km})$ for the complete journey of the train. The results of the rail potential and stray current are presented in Figs. 14 and 15, respectively. Unlike the grounded scheme, the rail potential and stray current now show negative values also. This is mainly because there is no intentional grounding at TSSs and the stray current follows the path of lower potential.

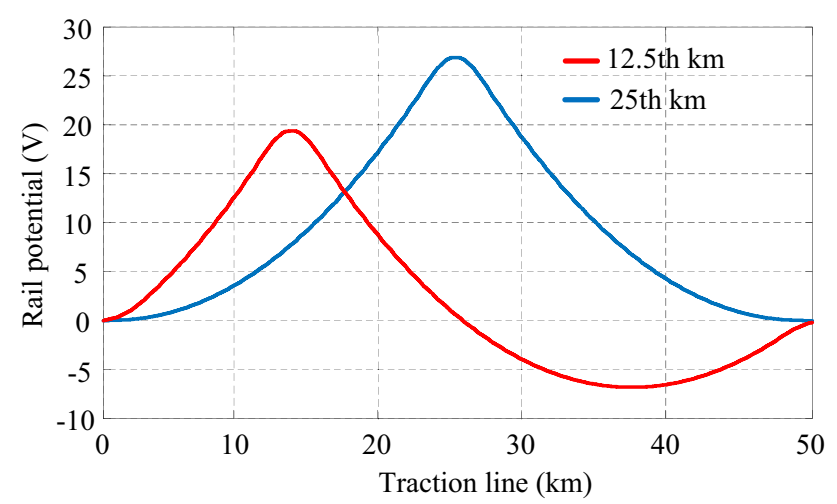

Fig. 14 Rail potential profile at specified locations for the $50 \mathrm{~km}$ floating track

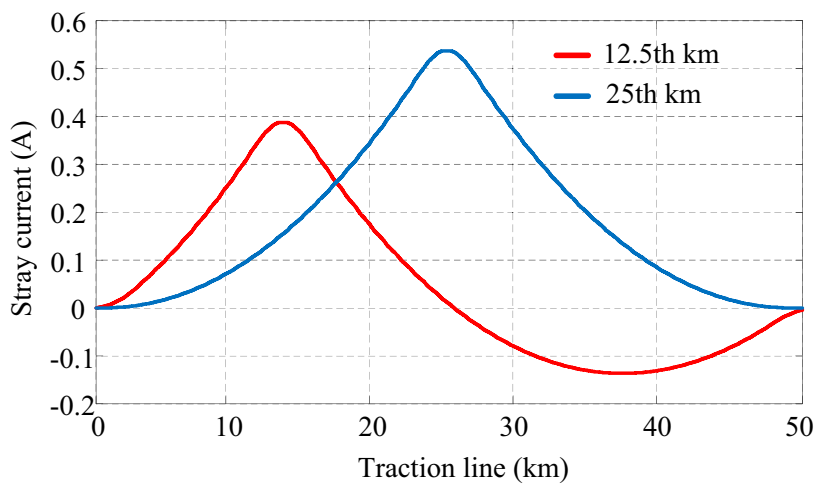

Fig. 15 Stray current profile at specified locations for the $50 \mathrm{~km}$ floating track

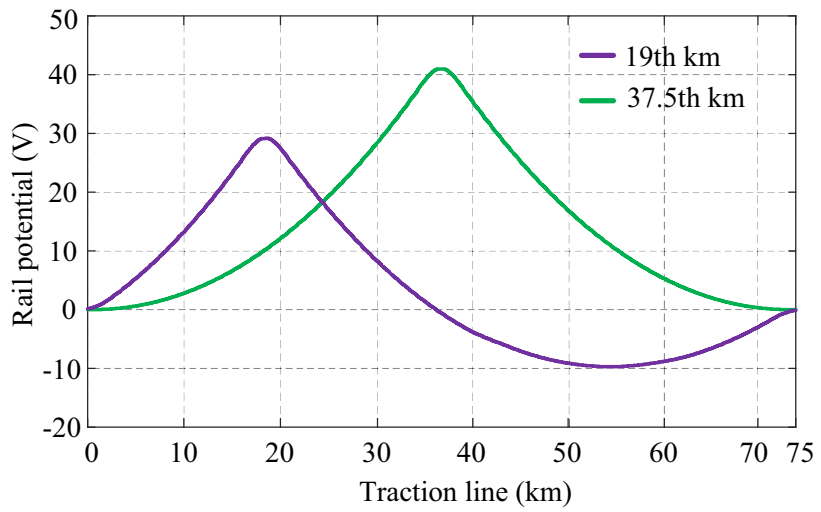

Fig. 16 Rail potential profile at specified locations for the $75 \mathrm{~km}$ floating track

\subsection{2 $75 \mathrm{~km}$ floating track}

Simulations are also performed for the proposed lengths of the MVDC-RES. The results of rail potential and stray current at the one-fourth $(19 \mathrm{~km})$ of the section and at midsection $(37.5 \mathrm{~km})$ are provided in Figs. 16 and 17. The results reveal that the values of stray current and rail potential for floating scheme are much lower as compared to the values obtained for grounded scheme track.

\subsubsection{Verification of simulations}

The simulation results of the rail potential and stray current depicted for the floating track can be verified through 


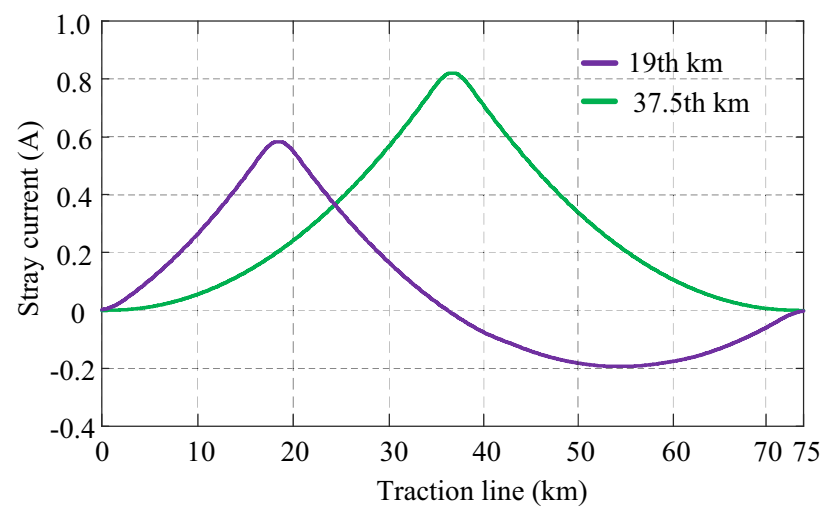

Fig. 17 Stray current profile at specified locations for the $75 \mathrm{~km}$ floating track

Eqs. (3) and (4), respectively. The verification is only performed for the complete profile of stray current at the one-fourth (19th km) of the $75 \mathrm{~km}$ track. Figure 18 shows a simplified version of Fig. 5 .

Given the train direction from left to right, initially when the train is in area A, the track current $I_{2}$ is responsible for rail potential and stray current at the one-fourth of the distance or at the 19 th $\mathrm{km}$ resistor. $I_{2}$ can be monitored through an ammeter $A_{a}$ and further used in Eq. (3) for the estimation of stray current at 19 th $\mathrm{km}$. As the train enters area $\mathrm{B}$, the track current $I_{1}$ will now be responsible for rail potential and stray current at the 19 th $\mathrm{km}$. $I_{1}$ can be monitored through an ammeter $A_{b}$ and used in Eq. (3) for the estimation of stray current at the 19th km of the track. The portions of track currents $I_{2}$ and $I_{1}$ that cause stray current at the 19th km are shown in Figs. 19 and 20.

Considering the values of current from the above figures, the stray current can be estimated at the 19 th $\mathrm{km}$ through Eq. (3) for the train positioned at 10, 19, 30, and $60 \mathrm{~km}$. In Table 4, the results are compared with the simulated values. It can be observed from Table 4 that estimated values agree with the simulation results.

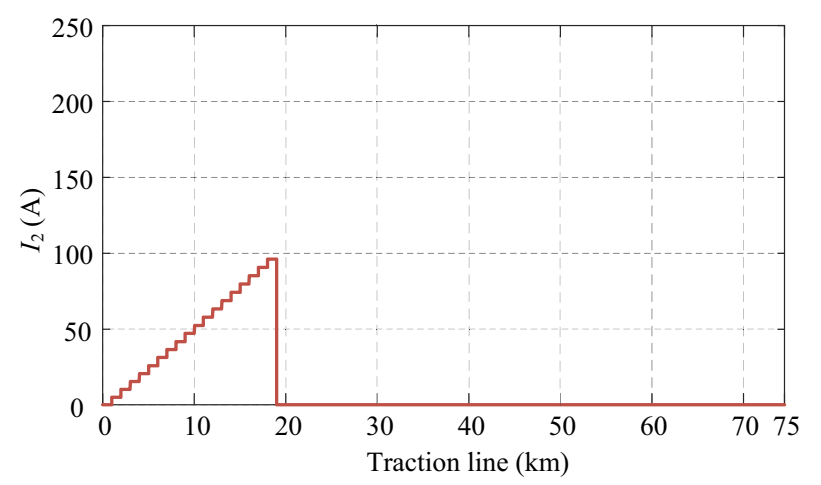

Fig. 19 Track current $I_{2}$ at the 19 th $\mathrm{km}$ for train journey in area A

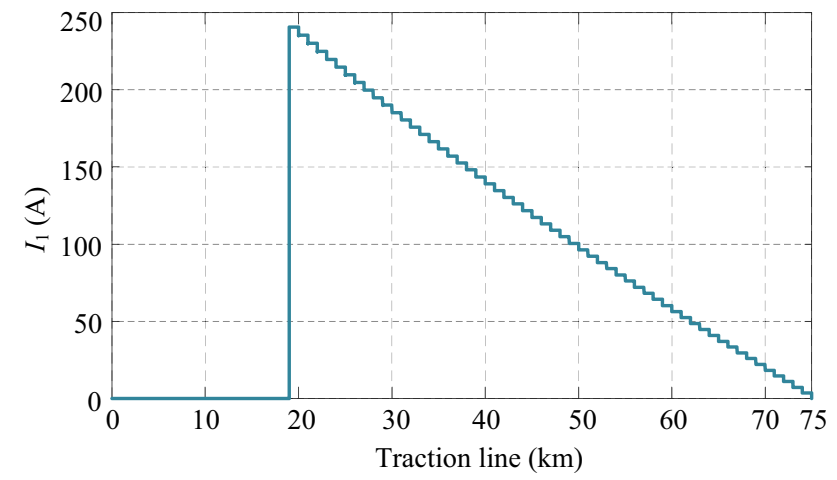

Fig. 20 Track current $I_{1}$ at the 19th km for train journey in area B

\subsection{Simulations for $300 \mathrm{~km}$ multi-train multi-TSS scenario}

The stray current in a grounded track increases to its maximum level when the train reaches at the mid-section between two adjacent TSSs. However, the behaviors of the stray current and rail potential must not be the same in the case of a multi-train multi-TSS scenario. For detailed analysis, simulations are performed for both grounded and floating schemes. To consider a more realistic simulation model, a $300 \mathrm{~km}$ track is considered, which resembles the newly build Chengdu-Chongqing long-distance highspeed line. Moreover, as per the timetable/schedule of the

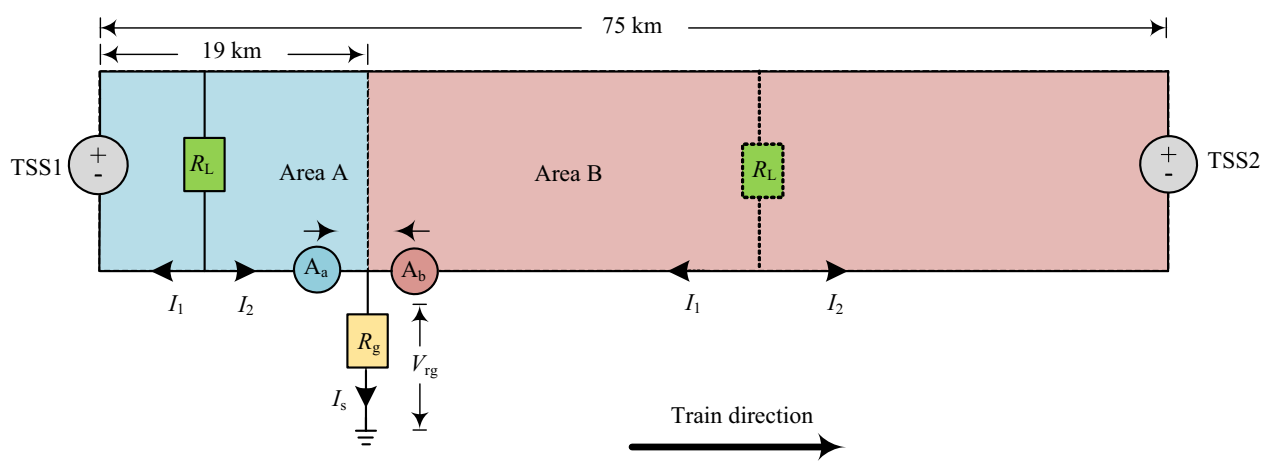

Fig. 18 A simplified model of Fig. 5 for verification of stray current for the $75 \mathrm{~km}$ floating track 
Table 4 Validation of stray current for floating track simulation

\begin{tabular}{|c|c|c|c|c|c|}
\hline Train location $(\mathrm{km})$ & $I_{2}(\mathrm{~A})$ & $I_{1}(\mathrm{~A})$ & Length considered $l(\mathrm{~km})$ & Estimated stray current through Eq. (3) (A) & Simulated stray current (A) \\
\hline 10 & 48 & - & 56 & 0.23 & 0.24 \\
\hline 19 & 95 & 243 & 19,56 & $0.48,0.55$ & 0.58 \\
\hline 30 & - & 180 & 19 & 0.36 & 0.19 \\
\hline 60 & - & 65 & 19 & -0.15 & -0.18 \\
\hline
\end{tabular}

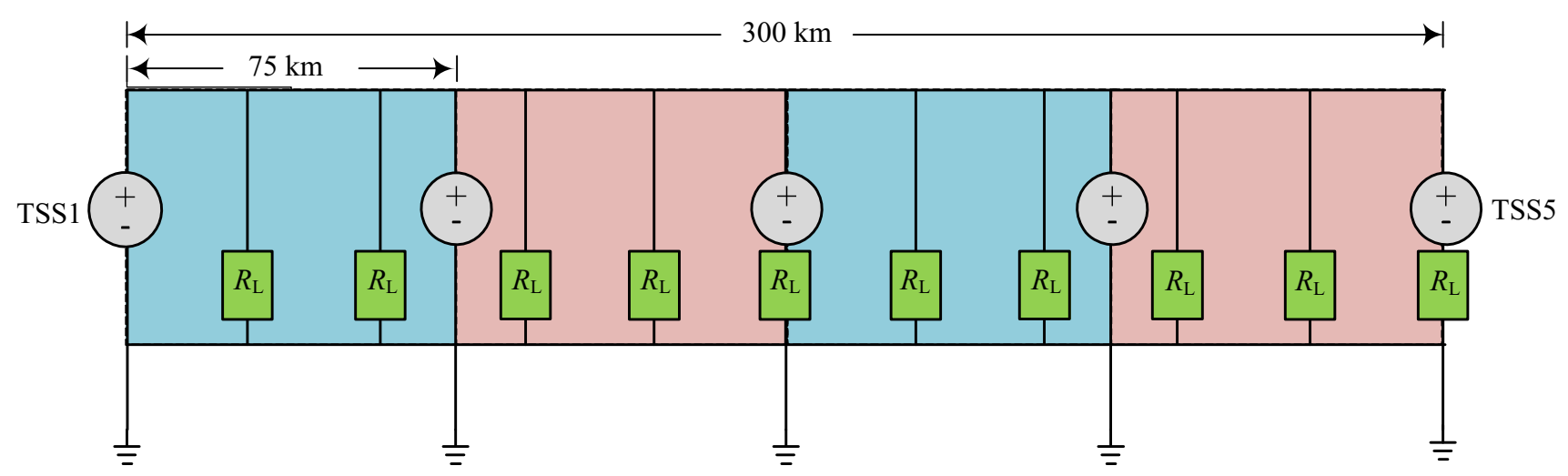

Fig. 21 Simulation scenario for a $300 \mathrm{~km}$ grounded track

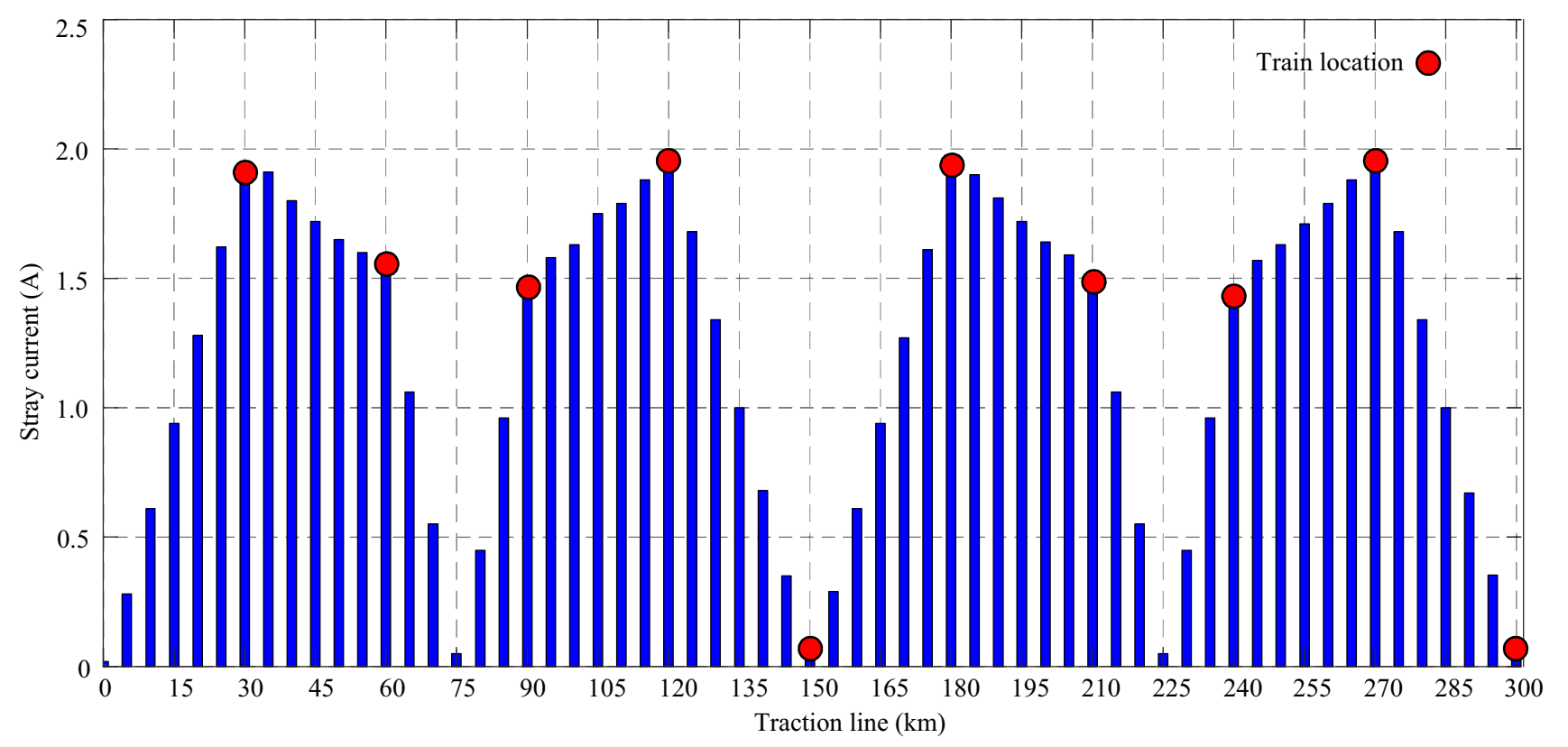

Fig. 22 Stray current profile for the $300 \mathrm{~km}$ grounded track

considered line, 10 active trains are considered in simulations.

\subsection{1 $300 \mathrm{~km}$ multi-train multi-TSS grounded track scheme}

For simulation, a $300 \mathrm{~km}$ grounded track is considered, which has five TSSs each at a distance of $75 \mathrm{~km}$ from each other. Ten trains are equally distributed, each at a distance of $30 \mathrm{~km}$ from the other. The combination of TSSs and trains is provided in Fig. 21.

The obtained stray current results are provided in Fig. 22, which is observed from 60 different locations along the track. It is found that the maximum stray current along the track is not more than $2 \mathrm{~A} / \mathrm{km}$, which is less than 


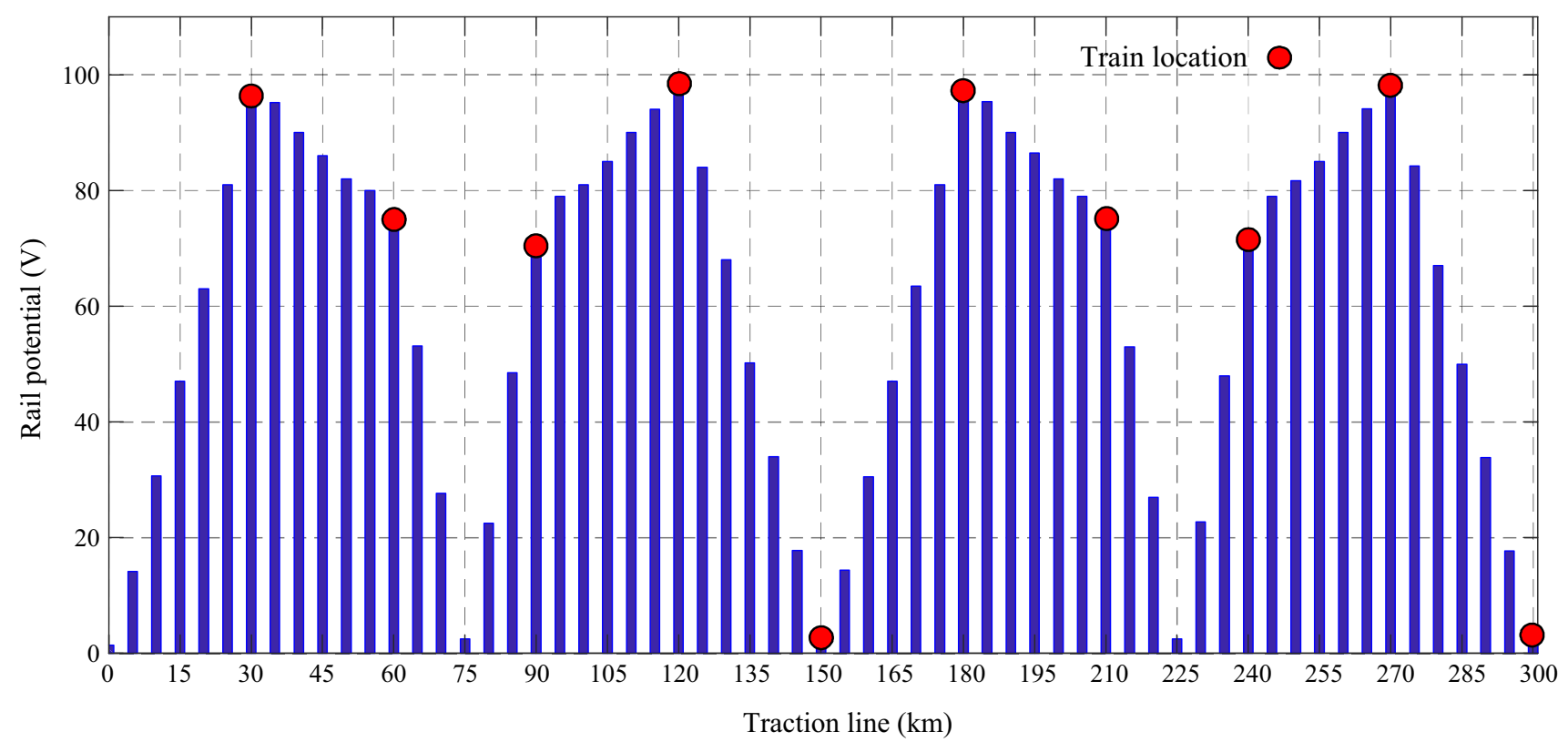

Fig. 23 Rail potential profile for the $300 \mathrm{~km}$ grounded track

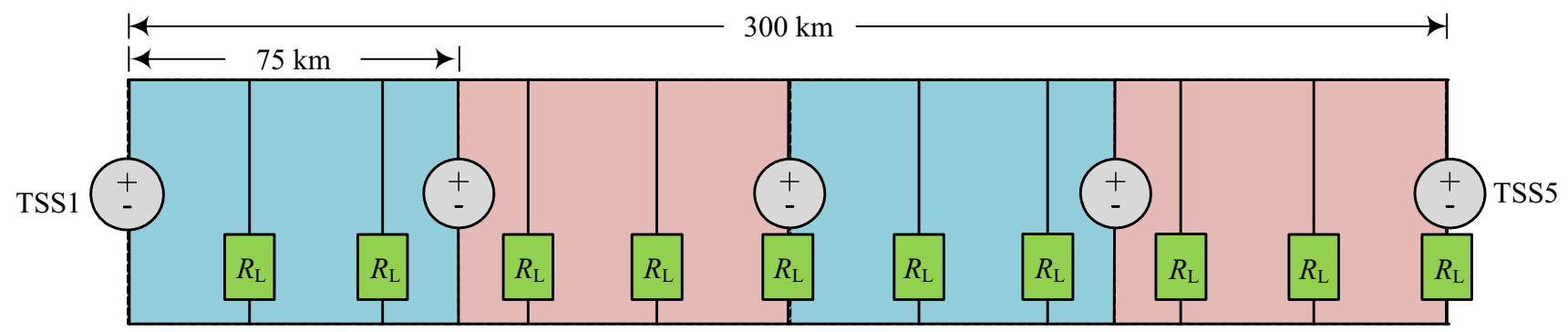

Fig. 24 Simulation scenario for the $300 \mathrm{~km}$ floating track

the averaged allowable value of $2.5 \mathrm{~A} / \mathrm{km}$. It is also noteworthy that the measured current is the total current leaking through the considered grounding resistance that further has to divide and flow toward TSSs. This division will depend upon the distance between the TSS and concerned track location; the more the distance between the TSS and train location, the less the stray current in that direction will be.

The simulated rail potential is provided in Fig. 23 . Although the stray current is within the limits, the rail potential slightly increases from the maximum limit of $90 \mathrm{~V} / \mathrm{km}$. Practically, a combination of grounded and floating scheme can be achieved through diode or thyristor grounding, which can considerably decrease the rail potential.

\subsection{2 $300 \mathrm{~km}$ multi-train multi-TSS floating scheme}

Simulation scenario for a floating scheme is given in Fig. 24. The model is same as discussed in previous section, where the only difference is that the ground terminals are removed.

The simulation result of stray current is provided in Fig. 25. Locations of TSSs and trains are same as discussed in previous section. It can be observed that the stray current is now reduced to a maximum value of about $1.5 \mathrm{~A} / \mathrm{km}$.

The profile of rail potential is shown in Fig. 26, revealing a considerable decrease in rail potential for floating scheme. The maximum value of rail potential is approximately $-70 \mathrm{~V}$ which is less than that of safe limits of $-90 \mathrm{~V}$. 


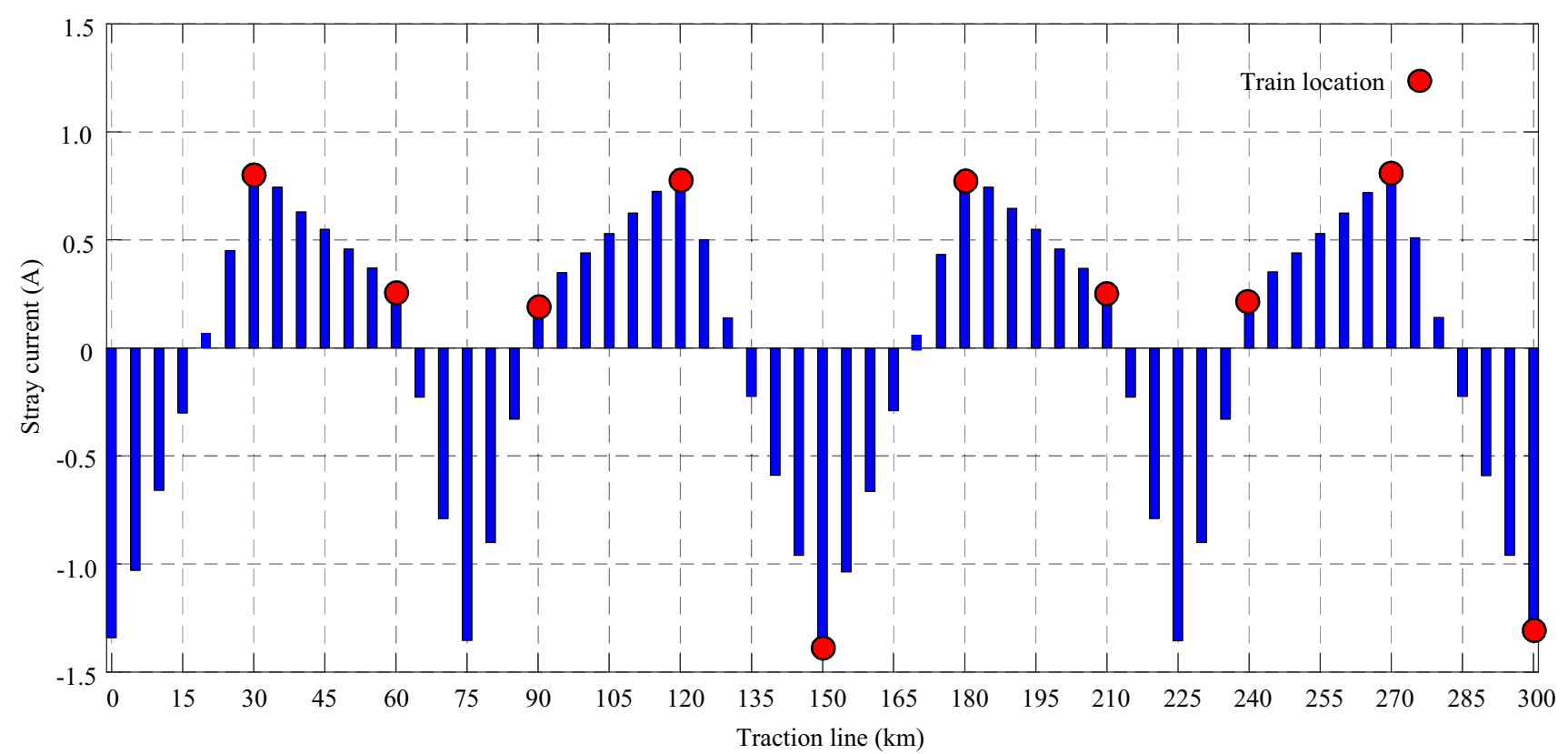

Fig. 25 Stray current profile for $300 \mathrm{~km}$ floating track

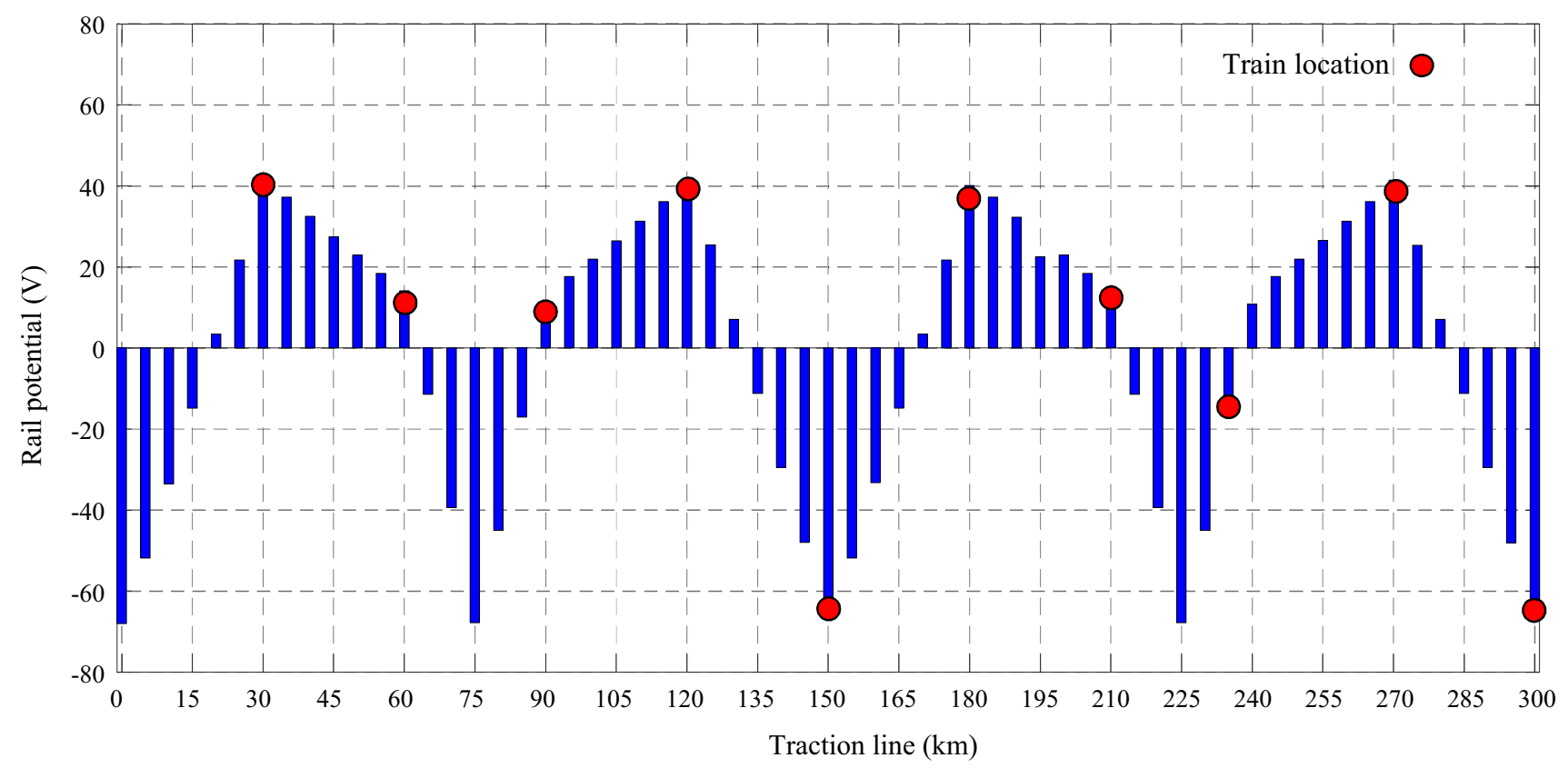

Fig. 26 Rail potential profile for the $300 \mathrm{~km}$ floating track

\subsection{Safe operation distances between adjacent TSSs in MVDC-RES}

Considering the constraints of rail potential and stray current, analysis is performed to determine the maximum safe distance between adjacent TSSs. For simulations, two trains, each of $8 \mathrm{MW}$, are placed at equal distance from each other as well as from the TSS. Simulations are performed with different track lengths in the grounded and floating schemes. For the grounded scheme, the results of rail potential and stray current are provided in Figs. 27 and 28 , respectively. The rail potential profile in grounded scheme depicts safe results for distances up to $70 \mathrm{~km}$. For the track lengths of $80 \mathrm{~km}$ and $90 \mathrm{~km}$, the rail potential reaches to hazardous levels. Unlike rail potential, stray current remains under safe limits even for the $90 \mathrm{~km}$ track.

Simulations results of rail potential and stray current for different tracks under the floating scheme are illustrated in 


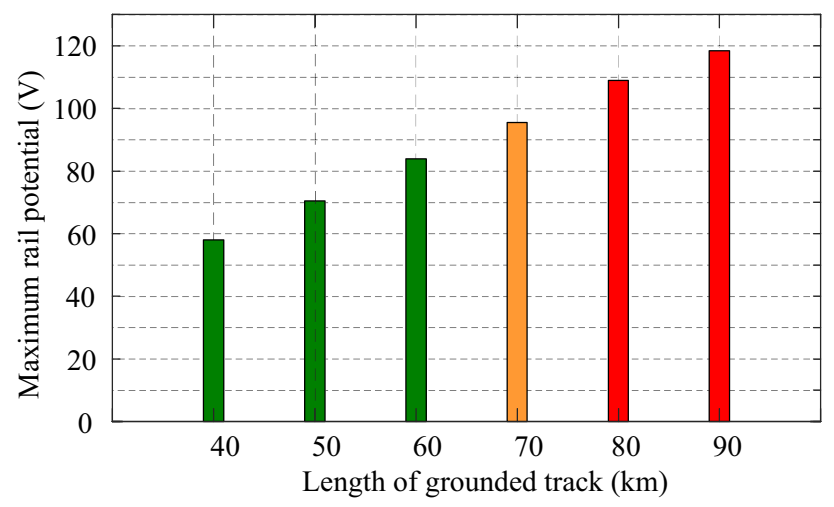

Fig. 27 Rail potential with different track lengths in the grounded scheme

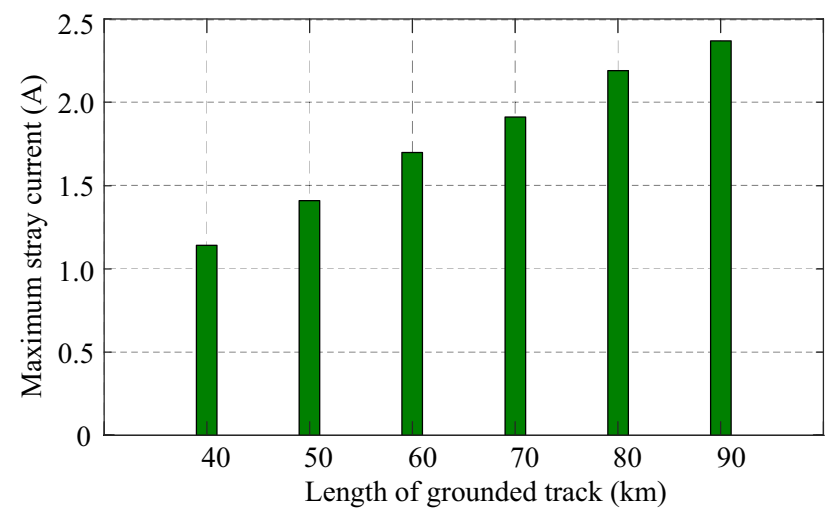

Fig. 28 Stray current for different track lengths in the grounded scheme

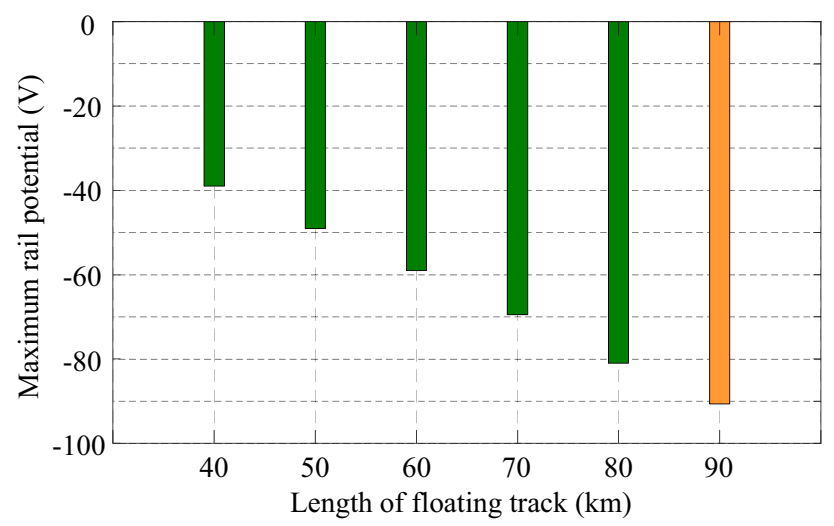

Fig. 29 Rail potential with different track lengths in the floating scheme

Figs. 29 and 30, respectively. Rail potential under the floating scheme depicts safe results for track lengths of up to $80-85 \mathrm{~km}$. Similarly, the stray current remains under safe values for the track lengths of up to $90 \mathrm{~km}$.

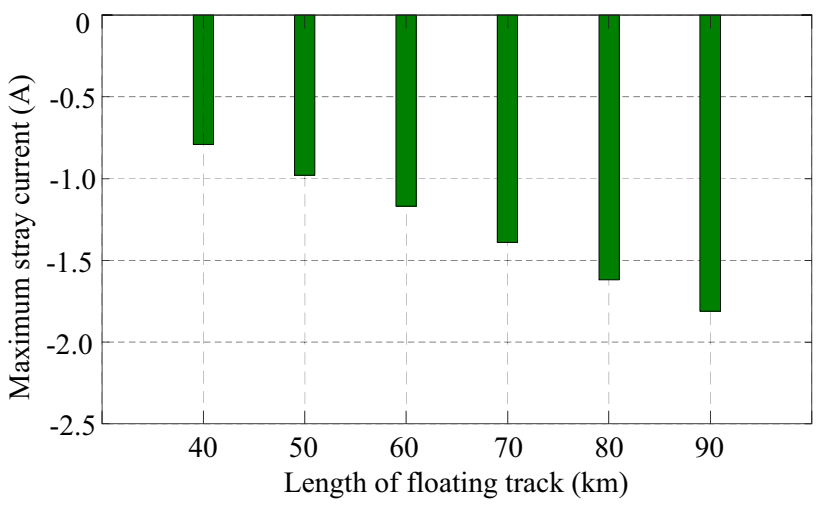

Fig. 30 Stray current with different track lengths in the floating scheme

\section{Conclusion}

The main purpose of this study is to analyze the behavior of rail potential and stray current in the MVDC railway electrification environment. A simulation model is proposed to find the profiles of rail potential and stray current in stationary and moving train scenarios. Simulations are performed for both the grounded and floating schemes. In the moving train scenario, the results of rail potential and stray current are monitored at different locations for the complete journey of the train. The results reveal that the rail potential and stray current change drastically with changing locations of the moving train. Simulations are also performed in a multi-train multi-substation scenario on a $300 \mathrm{~km}$ traction line, with TSSs spaced at a distance of $75 \mathrm{~km}$ from each other. The results demonstrate that the rail potential and stray current remains under safe values in the floating scheme; however, the rail potential in the grounded scheme increases up to the maximum threshold level. The results obtained are theoretically verified for the estimation of stray current. Results also indicate that train location is an important factor when analyzing a multi-train multi-substation scenario. A single train at the mid-section can cause more stray current as compared to the two trains near the substation. Furthermore, for the guidance of MVDC-RES projects, simulations are performed to obtain the results of rail potential and stray current for different track lengths. As a result, in the grounded scheme, a distance of $70 \mathrm{~km}$ can be considered as the maximum safe distance between adjacent TSSs, whereas in the floating scheme, a distance of $80 \mathrm{~km}$ can be considered as a safe distance between adjacent TSSs.

Open Access This article is licensed under a Creative Commons Attribution 4.0 International License, which permits use, sharing, adaptation, distribution and reproduction in any medium or format, as long as you give appropriate credit to the original author(s) and the source, provide a link to the Creative Commons licence, and indicate if changes were made. The images or other third party material in this 
article are included in the article's Creative Commons licence, unless indicated otherwise in a credit line to the material. If material is not included in the article's Creative Commons licence and your intended use is not permitted by statutory regulation or exceeds the permitted use, you will need to obtain permission directly from the copyright holder. To view a copy of this licence, visit http://creativecommons. org/licenses/by/4.0/.

\section{References}

1. Tan D (2016) Transportation electrification: challenges and opportunities. IEEE Power Electron Mag 3(2):50-52

2. Gómez-Expósito A, Mauricio JM, Maza-Ortega JM (2014) VSCbased MVDC railway electrification system. IEEE Trans Power Delivery 29(1):422-431

3. Yang X, Hu H, Ge Y, Aatif S, He Z, Gao S (2018) An improved droop control strategy for VSC-based MVDC traction power supply system. IEEE Trans Ind Appl 54(5):5173-5186

4. Aatif S, Yang X, Hu H, Maharjan SK, He Z (2021) Integration of $\mathrm{PV}$ and battery storage for catenary voltage regulation and stray current mitigation in MVDC railways. J Mod Power Syst Clean Energy 9(3):1-11

5. Aatif S, Hu H, Yang X, Ge Y, He Z, Gao S (2019) Adaptive droop control for better current-sharing in VSC-based MVDC railway electrification system. J Mod Power Syst Clean Energy 7(4):962-974

6. Zaboli A, Vahidi B, Yousefi S, Hosseini-Biyouki MM (2017) Evaluation and control of stray current in DC-electrified railway systems. IEEE Trans Veh Technol 66(2):974-980

7. Cotton I, Charalambous C, Aylott P, Ernst P (2005) Stray current control in DC mass transit systems. IEEE Trans Veh Technol 54(2):722-730

8. Pham KD, Thomas RS, Stinger WE (2001) Analysis of stray current, track-to-earth potentials and substation negative grounding in DC traction electrification system. In: Proceedings of the 2001 IEEE/ASME Joint Railroad Conference, Toronto, pp. 141-160.

9. Verdicchio A, Ladoux P, Caron H, Courtois C (2018) New medium-voltage DC railway electrification system. IEEE Trans Trans Electr 4(2):591-604

10. E. C. f. E. Standardization, EN 50122-1:2011: Railway applications-fixed installations: electrical safety, earthing and the return circuit - Part 1: Protective provisions against electric shock, British Standards Institution, 2011.

11. Ogunsola A, Mariscotti A, Sandrolini L (2012) Estimation of stray current from a DC-electrified railway and impressed potential on a buried pipe. IEEE Trans Power Delivery 27(4):2238-2246

12. E. C. f. E. Standardization, EN 50122-2:2010: Railway applications - fixed installations: electrical safety, earthing and the return circuit - Part 2: Provisions against the effects of stray currents caused by d.c. traction systems, British Standards Institution, 2008.

13. Paul D (2002) DC traction power system grounding. IEEE Trans Ind Appl 38(3):818-824

14. Bahra KS, Batty PG (1998) Earthing and bonding of electrified railways. In: International Conference on Developments in Mass Transit Systems, London, pp. 296-302.

15. Charalambous CA, Aylott P, Buxton D (2016) Stray current calculation and monitoring in DC mass-transit systems: interpreting calculations for real-life conditions and determining appropriate safety margins. IEEE Veh Technol Mag 11(2):24-31

16. Du G, Wang J, Jiang X, Zhang D, Yang L, Hu Y (2020) Evaluation of rail potential and stray current with dynamic traction networks in multitrain subway systems. IEEE Trans Trans Elect 6(2):784-796

17. Lin S, Yang H, Zhou Q, Wang A (2020) Evaluation and analysis model of stray current in the metro depot. IEEE Trans Trans Elect. https://doi.org/10.1109/TTE.2020.3035395

18. Lin S, Wang A, Liu M, Lin X, Zhou Q, Zhao L (2020) A multiple sections model of stray current of DC metro systems. IEEE Trans Power Delivery 36(3):1582-1593

19. Mariscotti A (2021) Electrical safety and stray current protection with platform screen doors in DC rapid transit. IEEE Trans Trans Elect. https://doi.org/10.1109/TTE.2021.3051102

20. Yang X, Xue H, Wang H, Zheng T (2018) Stray current and rail potential simulation system for urban rail transit. In: 2018 IEEE International Power Electronics and Application Conference and Exposition (PEAC), Shenzhen, pp 1-6

21. Ibrahem A, Elrayyah A, Sozer Y, Abreu AD (2015) DC railway system emulator for stray current and touch voltage prediction. In: IEEE Energy Conversion Congress and Exposition (ECCE), Montreal, pp. 1320-1326.

22. Pan P, Hu H, Yang X, Blaabjerg F, Wang X, He Z (2018) Impedance measurement of traction network and electric train for stability analysis in high-speed railways. IEEE Trans Power Electron 33(12):10086-10100

23. Zhu X, Hu H, Tao H, He Z, Kennel RM (2019) Stability prediction and damping enhancement for MVDC railway electrification system. IEEE Trans Ind Appl 55(6):7683-7698

24. Hossain E, Perez R, Nasiri A, Padmanaban S (2018) A comprehensive review on constant power loads compensation techniques. IEEE Access 6:33285-33305 